\title{
Common Fixed Point Theorems for Probabilistic Nearly Densifying Mappings
}

\author{
Aeshah Hassan Zakri, ${ }^{1}$ Sumitra Dalal, ${ }^{1}$ Sunny Chauhan, ${ }^{2}$ and Jelena Vujakovic ${ }^{3}$ \\ ${ }^{1}$ Department of Mathematics, Faculty of Science, Jazan University, Jazan, Saudi Arabia \\ ${ }^{2}$ Near Nehru Training Centre, H. No. 274, Nai Basti B-14, Bijnor-246701, Uttar Pradesh, India \\ ${ }^{3}$ Faculty of Sciences and Mathematics, University of Priština, Lole Ribara 29, 38220 Kosovska Mitrovica, Serbia
}

Correspondence should be addressed to Sumitra Dalal; mathsqueen_d@yahoo.com

Received 15 October 2014; Revised 28 December 2014; Accepted 28 December 2014

Academic Editor: Poom Kumam

Copyright (C) 2015 Aeshah Hassan Zakri et al. This is an open access article distributed under the Creative Commons Attribution License, which permits unrestricted use, distribution, and reproduction in any medium, provided the original work is properly cited.

The aim of this paper is to prove some coincidence and common fixed point theorems for probabilistic nearly densifying mappings in complete Menger spaces. Our results improve the results of Chamola et al. (1991), Dimri and Pant (2002), and Pant et al. (2004) and extend the results of Khan and Liu (1997) in the framework of probabilistic settings.

\section{Introduction and Preliminaries}

Banach contraction mapping principle is one of the most interesting and useful tools in applied mathematics. In recent years many generalizations of Banach contraction mapping principle have appeared. The notion of probabilistic metric spaces (in short PM-spaces) is a probabilistic generalization of metric spaces which are appropriate to carry out the study of those situations wherein distances are measured in the sense of distribution functions rather than nonnegative real numbers. The study of PM-spaces was initiated by Menger [1]. Since then, Schweizer and Sklar [2] enriched this concept and provided a new impetus by proving some fundamental results on this theme. The first result on fixed point theory in PMspaces was given by Sehgal and Bharucha-Reid [3] wherein the notion of probabilistic contraction was introduced as a generalization of the classical Banach fixed point principle in terms of probabilistic settings. Some recent fixed point results can be studied in [4-7].

Kuratowski [8] introduced the notion of measure of noncompactness of a bounded subset of a metric space. Further, this study was carried on by Furi and Vignoli [9]. They introduced the notion of densifying (also called condensing) mapping in terms of Kuratowski's measure of noncompactness and obtained some fixed point theorems. Following Furi and Vignoli [9], a number of mathematicians worked on densifying mappings and proved some metrical fixed point theorem (cf. [10-14]). As a generalization of Kuratowski's measure of noncompactness, Bocsan and Constantin [15] introduced the notion of Kuratowski's measure of noncompactness in PMspaces. Subsequently, Bocşan [16] studied the notion of probabilistic densifying mappings. Later, Hadžić [17], Tan [18], Chamola et al. [19], Dimri and Pant [20], Pant et al. [21], Pant et al. [22], and Singh and Pant [23] proved some results for such mappings. In [24], Ganguly et al. introduced the notion of probabilistic nearly densifying mappings and proved some interesting results in this setting.

The aim of this paper is to prove some coincidence and common fixed point theorems for certain classes of nearly densifying mappings in complete Menger spaces. First, we give some topological definitions and terminology defined in $[8,15-17]$.

Definition 1. A semigroup $G$ is said to be left reversible if for any $r, s \in G$ there exist $a, b \in G$ such that $r a=s b$.

It is easy to see that the notion of left reversibility is equivalent to the statement that any two right ideals of $G$ have nonempty intersection. 
Definition 2. Let $G$ be a family of self-mappings in $X$. A subset $Y$ of $X$ is called $G$-invariant if $g Y \subseteq Y$ for all $g \in G$.

Definition 3. Let $G^{*}$ be the semigroup generated by $G$ under composition *. Clearly, $G^{*} \supseteq\left\{g^{n}: n \geq 0\right\}$ for any $g \in G$ and $G^{*}(u)=\{u\} \cup\left\{g u: g \in G^{*}\right\}$ for $u \in X$.

We restate the notion of probabilistic diameter for the sake of quick reference.

Definition 4. Let $A$ be a nonempty subset of $X$. A function $D_{A}(\cdot)$ defined by

$$
D_{A}(x)=\sup _{y<x}\left\{\inf _{u, v \in A} F_{u, v}(y)\right\}
$$

is called probabilistic diameter of $A$. $A$ is said to be bounded if

$$
\sup _{x \in R} D_{A}(x)=1 .
$$
[15].

The following definition is due to Bocsan and Constantin

Definition 5. For a probabilistic bounded subset $A$ of $X$, $\alpha_{A}(x)$ defined by $\alpha_{A}(x)=\sup \{\varepsilon \geq 0: \exists$ a finite cover $\mathscr{A}$ of $A$ such that $D_{S}(x) \geq \varepsilon$ for all $\left.S \in \mathscr{A}\right\}$ is called Kuratowski's function.

The following properties of Kuratowski's functions are proved in [8]:

(a) $\alpha_{A} \in \mathfrak{I}$, the set of distribution functions;

(b) $\alpha_{A}(x) \geq D_{A}(x)$;

(c) if $\phi \neq A \subset B \subset X$, then $\alpha_{A}(x) \geq \alpha_{B}(x)$;

(d) $\alpha_{A \cup B}(x)=\min \left\{\alpha_{A}(x), \alpha_{B}(x)\right\}$;

(e) let $\bar{A}$ be the closure of $A$ in the $(\varepsilon, \lambda)$-topology on $X$; then

$$
\alpha_{\bar{A}}(x)=\alpha_{A}(x)
$$

(f) $A$ is probabilistic precompact (totally bounded) if $\alpha_{A}=H$,

where $H$ denotes the specific distribution function defined by

$$
H(x)= \begin{cases}0, & x \leq 0 \\ 1, & x>0\end{cases}
$$

Definition 6. Let $(X, \mathscr{F})$ be a PM-space. A continuous mapping $f$ of $X$ into $X$ is called a probabilistic densifying mapping if and only if, for every subset $A$ of $X, \alpha_{A}<H$ implies $\alpha_{f(A)}>\alpha_{A}$.

Definition 7. A self-mapping $f: X \rightarrow X$ is probabilistic nearly densifying if $\alpha_{f(A)}>\alpha_{A}$, whenever $\alpha_{A}<H, A \subset H$, and $A$ is $f$-invariant.

Definition 8. Suppose $\phi:[0, \infty) \rightarrow[0, \infty)$ is an upper semicontinuous function with $\phi(0)=0$ and $\phi(t)<t$ for all $t>0$.

\section{Main Results}

First, we prove some fixed point theorems for probabilistic nearly densifying mappings in Menger spaces.

Theorem 9. Let $P, Q$, and $R$ be three continuous and nearly densifying self-mappings on a complete Menger space $(X, \mathscr{F}, *)$ such that $\sup x * x=1$ and $R$ commutes with $P$ and $Q$. If, for all $x<1, u, v \in X$, the following conditions are satisfied:

$$
\begin{gathered}
\phi_{1}(P u, Q v)>\min \left\{\phi_{2}(R u, R v), \phi_{2}(R u, P u),\right. \\
\left.\phi_{1}(R v, Q v), \frac{\phi_{2}(R u, P u) \phi_{1}(R v, Q v)}{\phi_{2}(R u, R v)}\right\} \\
\text { for } R u \neq R v, \quad P u \neq Q v ; \\
\phi_{2}(Q u, P v)>\min \left\{\phi_{1}(R u, R v), \phi_{1}(R u, Q u), \phi_{2}(R v, P v),\right. \\
\left.\frac{\left.\phi_{1}(R u, Q u) \phi_{2}(R v, P v)\right\},}{\phi_{1}(R u, R v)}\right\} \\
\text { for } R u \neq R v, \quad Q u \neq P v,
\end{gathered}
$$

where $\phi_{1}$ and $\phi_{2}$ are real valued mappings from $X \times X$ to $\varsigma$, the collection of all distribution functions, with either $\phi_{1}$ or $\phi_{2}$ being upper semicontinuous (u.s.c.) and $\phi_{1}(u, u)=\phi_{2}(u, u)=1$ for all $u \in X$.

Further, if, for some $u_{0} \in X, G\left(u_{0}\right)=\left\{P^{i} Q^{j} R^{k} u_{0}: i=\right.$ $0,1,2, \ldots ; j=0,1,2, \ldots ; k=0,1,2, \ldots\}$ is bounded, then $P$ and $R$ or $Q$ and $R$ have a coincidence point.

Proof. For $u_{0} \in X$, let $A=G\left(u_{0}\right)$ and $S=\{P Q R\}$.

Then $A=\left\{u_{0}\right\} \cup P(A) \cup Q(A) \cup R(A)$.

If $\alpha_{A}<H$, then

$$
\begin{aligned}
\alpha_{A} & =\alpha_{\left\{u_{0}\right\} \cup P(A) \cup Q(A) \cup R(A)} \\
& =\min \left\{\alpha_{P(A)}, \alpha_{Q(A)}, \alpha_{R(A)}\right\}>\alpha_{A},
\end{aligned}
$$

a contradiction. It implies that $\bar{A}$ is precompact.

Let $B=\bigcap_{n=0}^{\infty}(P Q R)^{n}(\bar{A})$.

Then it is easy to see that $S B=B$ and $B$ is nonempty compact subset of $A$. By the continuity of $P, Q$, and $R$, it follows that $P \bar{A} \subset \bar{A}, Q \bar{A} \subset \bar{A}$, and $R \bar{A} \subset \bar{A}$. Further, it is clear that $P(\bar{B}) \subset \bar{B}, Q(\bar{B}) \subset \bar{B}$, and $R(\bar{B}) \subset \bar{B}$.

Note that

$$
\begin{aligned}
R(B) & =\bigcap_{n=0}^{\infty} R(P Q R)^{n}(\bar{A}) \subset \bigcap_{n=0}^{\infty}(P Q R)^{n} R(\bar{A}) \subset B, \\
B & =P Q R(B)=R P Q(B) \subset R P(B) \subset R(B),
\end{aligned}
$$

which implies $R(B)=B$ or $R^{2}(B)=B$.

Now, assume that $\phi_{1}$ is upper semicontinuous. Then the function $T: B \rightarrow \mathfrak{I}$, defined by $T(u)=\phi_{1}(R u, Q u)$, is u.s.c. So $T$ assumes its maximal value at some point $p$ in $B$. Clearly, 
$p \in R^{2}(B)$, so there is a $w \in B$ such that $p=R^{2}(w)$. Suppose that neither $P$ and $R$ nor $Q$ and $R$ have a coincidence point. Then

$$
\begin{gathered}
T(P Q(w)) \\
=\phi_{1}(R P Q(w), Q P Q(w)) \\
=\phi_{1}(P R Q(w), Q P Q(w)) \text { by }(5), \\
>\min \left\{\phi_{2}\left(R^{2} Q(w), R P Q(w)\right),\right. \\
\phi_{2}\left(R^{2} Q(w), P R Q(w)\right), \phi_{1}(R P Q(w), Q P Q(w)), \\
\left.\quad \frac{\phi_{2}\left(R^{2} Q(w), P R Q(w)\right) \phi_{1}(R P Q(w), Q P Q(w))}{\phi_{2}\left(R^{2} Q(w), R P Q(w)\right)}\right\} \\
=\phi_{2}\left(Q R^{2}(w), P R Q(w)\right), \text { by }(6), \\
\quad \phi_{2}\left(R^{2} Q(w), P R Q(w)\right), \\
\left.\quad \frac{\phi_{1}\left(R R^{2}(w), Q R^{2}(w)\right) \phi_{2}\left(R^{2} Q(w), P R Q(w)\right)}{\phi_{1}\left(R R^{2}(w), R^{2} Q(w)\right)}\right\} \\
=\phi_{1}\left(R R^{2}(w), Q R^{2}(w)\right)=\phi_{1}(R p, Q p)=T(p),
\end{gathered}
$$

a contradiction to the selection of $p$. Hence, $P$ and $R$ or $Q$ and $R$ must have a coincidence point.

The same result holds good if $\phi_{2}$ is upper semicontinuous. This completes the proof of the theorem.

Remark 10. The above theorem extends the results of Khan and Liu [25, Theorem 3.1 and Corollary 3.3] to PM-spaces.

Theorem 11. Let $X, P, Q$, and $R$ be as in Theorem 9. Further, let $P, Q$, and $R$ satisfying (5) and (6) have a coincidence point $w$; then $R w$ is a unique common fixed point of $P, Q$, and $R$.

Proof. We have $P w=Q w=R w$. By commutativity of $R$ with $P$ and $Q, P R(w)=R P(w)=R R(w)$ and $Q R(w)=R Q(w)=$ $R R(w)$, or $P R(w)=R R(w)=Q R(w)$.

Now let $R^{2} w \neq R w$; then by (5) and (6), we have

$$
\begin{aligned}
& \phi_{1}\left(R^{2} w, R w\right) \\
&=\phi_{1}(P R w, Q w) \\
&> \min \left\{\phi_{2}\left(R^{2} w, R w\right), \phi_{2}\left(R^{2} w, P R w\right),\right. \\
&\left.\phi_{1}(R w, Q w), \frac{\phi_{2}\left(R^{2} w, P R w\right) \phi_{1}(R w, Q w)}{\phi_{2}\left(R^{2} w, R w\right)}\right\}
\end{aligned}
$$

$$
\begin{aligned}
& =\phi_{2}\left(R^{2} w, P R w\right)=\phi_{2}(Q R w, P w) \\
& >\min \left\{\phi_{1}\left(R^{2} w, R w\right), \phi_{1}\left(R^{2} w, Q R w\right),\right. \\
& \left.\phi_{2}(R w, P w), \frac{\phi_{1}\left(R^{2} w, Q R w\right) \phi_{2}(R w, P w)}{\phi_{1}\left(R^{2} w, R w\right)}\right\} \\
& =\phi_{1}\left(R^{2} w, R w\right),
\end{aligned}
$$

which is a contradiction. Hence, $R^{2} w=R w$. Thus, $R w$ is a fixed point of $R$. Thus, $R w=R(R w)=P(R w)=Q(R w)$. Therefore, $R w$ is a common fixed point of $P, Q$, and $R$.

The uniqueness of $R w$ as a common fixed point of $P, Q$, and $R$ follows from (5) and (6).

Theorem 12. Let $f$ and $g$ be commuting, continuous, and nearly densifying self-mappings on a complete Menger space X satisfying

$$
\phi(g u, g v)>\min \{\phi(f u, f v), \phi(f u, g u), \phi(f v, g v)\}
$$

for $f u \neq f v, g u \neq g v$, and $u, v \in X$, where $\phi: X \times X \rightarrow \zeta$ is u.s.c. and $\phi(u, u)=1,, u \in X$. If, for some $u_{0}$ in $X, G\left(u_{0}\right)=$ $\left\{f^{i} g^{j} u_{0}: i=0,1,2, \ldots ; j=0,1,2, \ldots\right\}$ is bounded, then $f$ and $g$ have a unique common fixed point.

Proof. Let $A=G\left(u_{0}\right)$. Since $f$ and $g$ are commuting and continuous, we have $f(\bar{A}) \subseteq \bar{A}$ and $g(\bar{A}) \subseteq \bar{A}$ and then $A=$ $\left\{u_{0}\right\} \cup f(A) \cup g(A)$.

If $\alpha_{A}<H$, then

$$
\begin{aligned}
\alpha_{A} & =\alpha_{\left\{u_{0}\right\} \cup f(A) \cup g(A)} \\
& =\min \left\{\alpha_{f(A)}, \alpha_{g(A)}\right\}>\alpha_{A},
\end{aligned}
$$

which is a contradiction. It implies that $\bar{A}$ is precompact.

Now define $B=\bigcap_{n=0}^{\infty}(f g)^{n}(\bar{A})$. Since $\left\{(f g)^{n} A\right\}$ is a decreasing sequence of nonempty compact subset of $A$, it follows that $B$ is nonempty set such that $f(\bar{B}) \subset \bar{B}, g(\bar{B}) \subset \bar{B}$.

Suppose that $u \in B$; then $u \in(f g)^{n+1} \bar{A}$ for all $n$. Hence, there exists $\left\{x_{n}\right\} \subseteq(f g)^{n} \bar{A}$. Since $(f g)^{n} \bar{A}$ is compact and closed for all $n, f$ and $g$ are continuous and nearly densifying; therefore, there exists a point $p \in(f g)^{n} \bar{A}$ for all $n$ so that $f g(p)=u$. Hence, $u \in f(B)$ and $u \in g(B)$. Thus, we have

$$
f(B)=B=g(B) .
$$

Let us define a real valued function $\psi$ on $B$ by $\psi(u)=$ $\phi(f u, g u)$. It is u.s.c. and hence attains its maximum at some point $p \in B$. Then there exists a $w \in B$ such that $p=f w$. 
Suppose that there is no point $u$ in $X$ such that $f u=g u$; then we have by (11)

$$
\begin{aligned}
& \psi(g w) \\
& \quad=\phi(f g w, g g w)=\phi(g f w, g g w) \\
& \quad>\min \left\{\phi\left(f^{2} w, f g w\right), \phi\left(f^{2} w, g f w\right), \phi(f g w, g g w)\right\} \\
& \quad=\min \left\{\phi\left(f^{2} w, f g w\right), \phi(f g w, g g w)\right\} \\
& \quad=\phi\left(f^{2} w, f g w\right)=\phi(f p, g p)=\psi(p),
\end{aligned}
$$

which is a contradiction to the selection of $p$. Hence, there exists a $w_{0} \in B$ such that $f w_{0}=g w_{0}$ or $f^{2} w_{0}=f g w_{0}=g f w_{0}$.

Suppose $f^{2} w_{0} \neq f w_{0}$; then we have

$$
\begin{aligned}
\phi & \left(f^{2} w_{0}, f w_{0}\right) \\
& =\phi\left(g f w_{0}, g w_{0}\right) \\
& >\min \left\{\phi\left(f^{2} w_{0}, f w_{0}\right), \phi\left(f^{2} w_{0}, g f w_{0}\right), \phi\left(f w_{0}, g w_{0}\right)\right\} \\
& =\phi\left(f^{2} w_{0}, f w_{0}\right)
\end{aligned}
$$

which is a contradiction. Hence, $f^{2} w_{0}=g f w_{0}=f w_{0}$. Therefore, $f w_{0}$ is common fixed point of $f$ and $g$. Now we will prove the uniqueness of $f w_{0}$. Let $w$ be the other fixed point of $f$ and $g$; then, by (11), we have

$$
\begin{aligned}
& \phi\left(w, f w_{0}\right) \\
& \quad=\phi\left(g w, f g w_{0}\right)=\phi\left(g w, g f w_{0}\right) \\
& \quad>\min \left\{\phi\left(f w, f^{2} w_{0}\right), \phi(f w, g w), \phi\left(f^{2} w_{0}, g f w_{0}\right)\right\} \\
& \quad=\phi\left(f w, f^{2} w_{0}\right)=\phi\left(w, f w_{0}\right), \text { a contradiction. }
\end{aligned}
$$

Hence, $f w_{0}$ is unique. This completes the proof of the theorem.

Remark 13. Theorems 9, 11, and 12 improve the result of Chamola et al. [19], Dimri and Pant [20], Ganguly et al. [24], and Pant et al. [21] under more natural conditions.

\section{Conflict of Interests}

The authors declare that there is no conflict of interests regarding the publication of this paper.

\section{Authors' Contribution}

All authors contributed equally to this paper. The guidance of Aeshah Hassan Zakri is very important and she helped in revising the paper according to reviewers reports.

\section{Acknowledgment}

The authors thank the anonymous referees for their careful reading and useful suggestions on the paper. The authors are highly thankful to Deanship of Scientific Research, Jazan University, for financial support of this paper.

\section{References}

[1] K. Menger, "Statistical metrics," Proceedings of the National Academy of Sciences of the United States of America, vol. 28, pp. 535-537, 1942.

[2] B. Schweizer and A. Sklar, "Statistical metric spaces," Pacific Journal of Mathematics, vol. 10, pp. 313-334, 1960.

[3] V. M. Sehgal and A. T. Bharucha-Reid, "Fixed points of contraction mappings on probabilistic metric spaces," Mathematical Systems Theory, vol. 6, pp. 97-102, 1972.

[4] W. Sintunavarat and P. Kumam, "Some fixed point results for weakly isotone mappings in ordered Banach spaces," Applied Mathematics and Computation, vol. 224, pp. 826-834, 2013.

[5] S. Chauhan, S. Dalal, W. Sintunavarat, and J. Vujakovíc, "Common property (E.A) and existence of fixed points in Menger spaces," Journal of Inequalities and Applications, vol. 2014, no. 1, article 56, 2014.

[6] S. Chauhan, M. Imdad, C. Vetro, and W. Sintunavarat, "Hybrid coincidence and common fixed point theorems in Menger probabilistic metric spaces under a strict contractive condition with an application," Applied Mathematics and Computation, vol. 239, pp. 422-433, 2014.

[7] A. Roldán-López-de-Hierro and W. Sintunavarat, "Common fixed point theorems in fuzzy metric spaces using the CLRg property," Fuzzy Sets and System, 2014.

[8] C. Kuratowski, "Sur les espaces complets," Fundamenta Mathematicae, vol. 15, pp. 301-309, 1930.

[9] M. Furi and A. Vignoli, "Fixed points for densifying mappings," Accademia Nazionale dei Lincei, vol. 47, pp. 465-467, 1969.

[10] B. Fisher and M. S. Khan, "Results on fixed points of densifying mappings," Mathematics Seminar Notes. Kobe University, vol. 7, pp. 509-514, 1969.

[11] R. K. Jain, "Some common fixed point theorems for nearly densifying maps," Journal of the Indian Academy of Mathematics, vol. 13, no. 1, pp. 12-15, 1991.

[12] R. D. Nussbaum, "The fixed point index for local condensing maps," Annali di Matematica Pura ed Applicata: Serie Quarta, vol. 89, pp. 217-258, 1971.

[13] S. Park, "On densifying maps of metric spaces," Honam Mathematical Journal, vol. 3, pp. 23-30, 1981.

[14] K. P. R. Sastry and S. V. R. Naidu, "Fixed point theorems for nearly-densifying maps," The Nepali Mathematical Sciences Report, vol. 7, pp. 41-44, 1982.

[15] G. Bocsan and G. Constantin, "The Kuratowski function and some applications to the probabilistic metric spaces," Atti della Accademia Nazionale dei Lincei. Rendiconti. Classe di Scienze Fisiche, Matematiche e Naturali, vol. 55, no. 8, pp. 236-240, 1973.

[16] G. Bocşan, "On some fixed point theorems in probabilistic metric spaces," Mathematica Balkanica, vol. 4, pp. 67-70, 1974.

[17] O. Hadžić, "Fixed point theorems for multivalued mappings in probabilistic metric spaces," Fuzzy Sets and Systems, vol. 88, no. 2, pp. 219-226, 1997. 
[18] D. H. Tan, “On probabilistic condensing mappings," Revue Roumaine de Mathématique Pures et Appliquées, vol. 26, no. 10, pp. 1305-1317, 1981.

[19] K. P. Chamola, B. D. Pant, and S. L. Singh, "Common fixed point theorems for probabilistic densifying mappings," Mathematica Japonica, vol. 36, no. 4, pp. 769-775, 1991.

[20] R. C. Dimri and B. D. Pant, "Fixed points of probabilistic densifying mappings," Journal of Natural \& Physical Sciences, vol. 16, no. 1-2, pp. 69-76, 2002.

[21] B. D. Pant, R. C. Dimri, and V. B. Chandola, "Some results on fixed points of probabilistic densifying mappings," Bulletin of the Calcutta Mathematical Society, vol. 96, no. 3, pp. 189-194, 2004.

[22] B. D. Pant, B. M. L. Tiwari, and S. L. Singh, "Common fixed point theorems for densifying mappings in probabilistic metric spaces," Honam Mathematical Journal, vol. 5, pp. 151-154, 1983.

[23] S. L. Singh and B. D. Pant, "Common fixed points of a family of mappings in Menger and uniform spaces," Rivista di Matematica della Università di Parma: Serie IV, vol. 14, no. 4, pp. 8185, 1988.

[24] A. Ganguly, A. S. Rajput, and B. S. Tuteja, "Fixed points of probabilistic densifying mappings," Indian Academy of Mathematics: Journal, vol. 13, no. 2, pp. 110-114, 1991.

[25] M. S. Khan and Z. Q. Liu, "On coincidence points of densifying mappings," Turkish Journal of Mathematics, vol. 21, no. 3, pp. 269-276, 1997. 


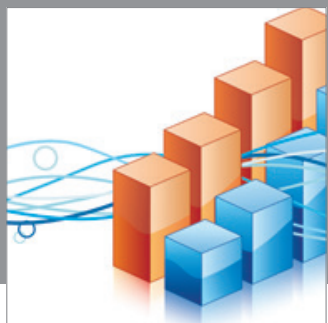

Advances in

Operations Research

mansans

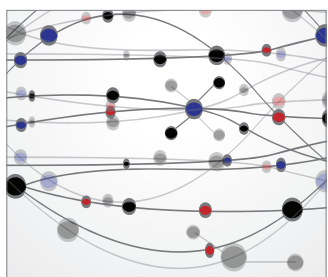

The Scientific World Journal
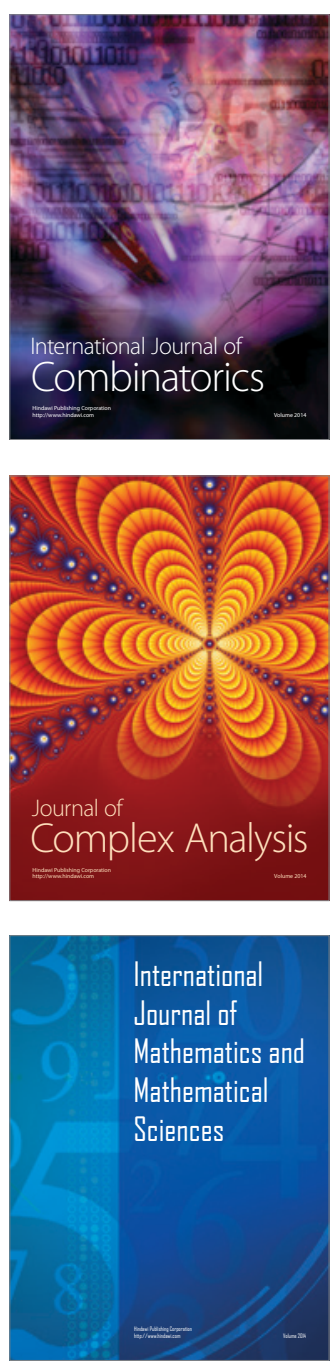
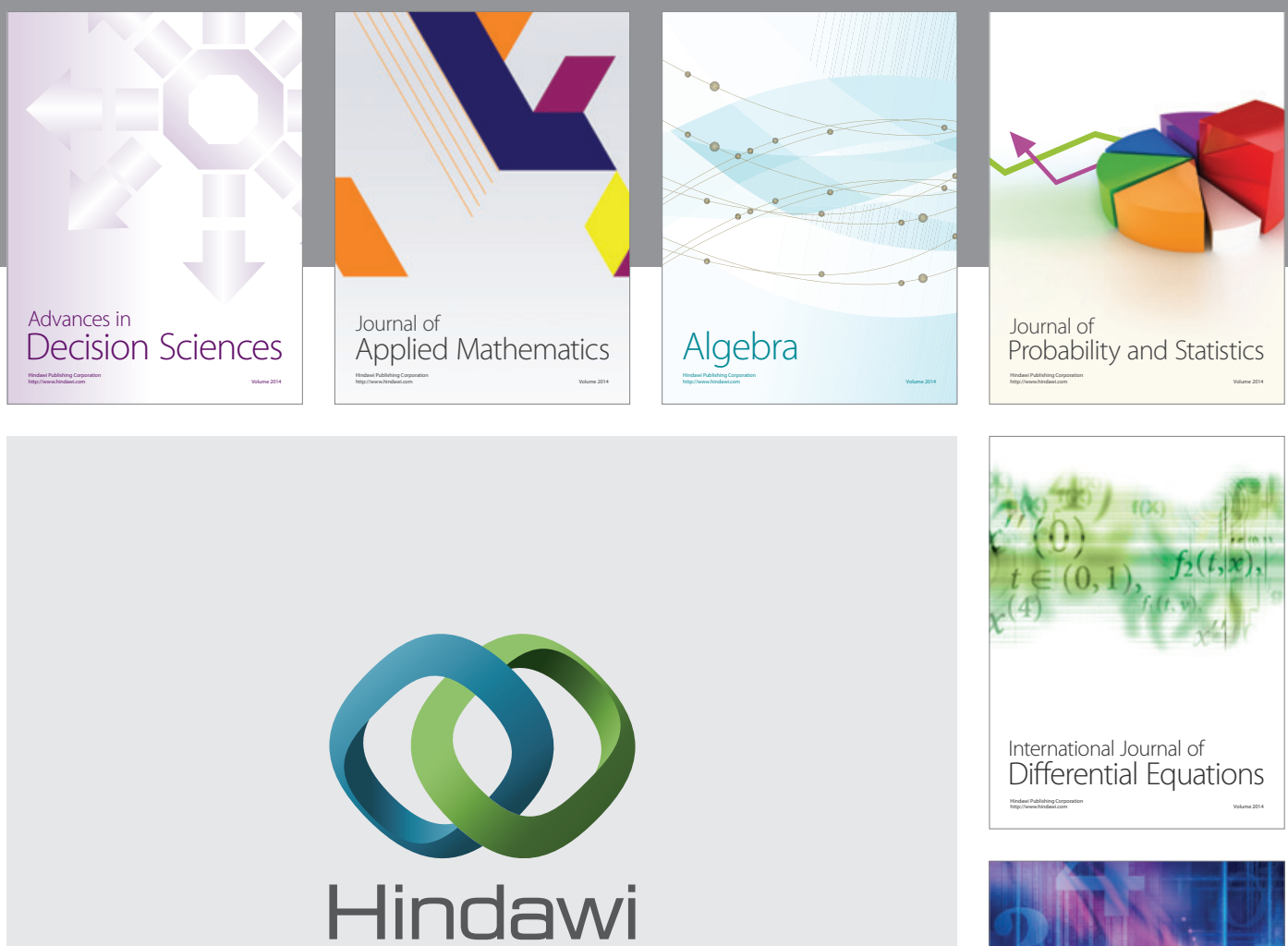

Submit your manuscripts at http://www.hindawi.com
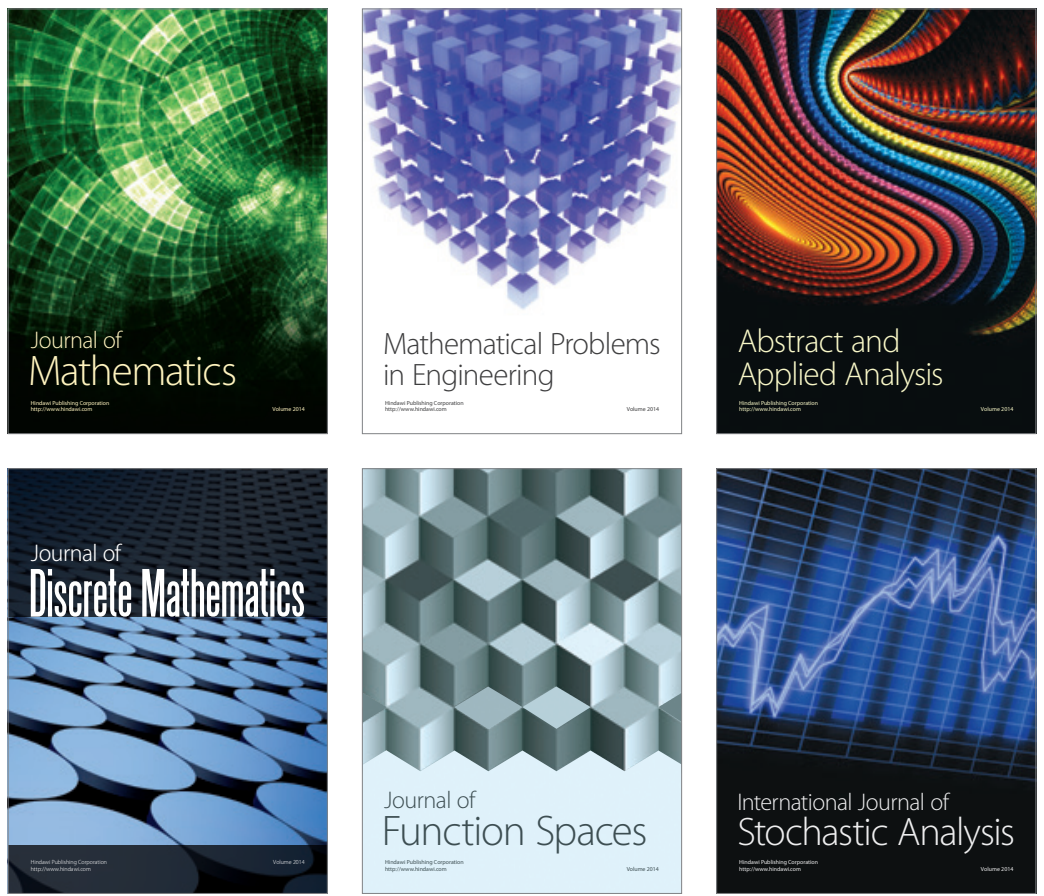

Journal of

Function Spaces

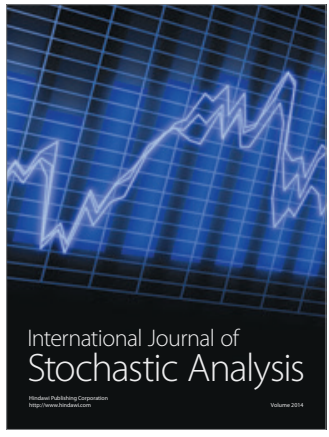

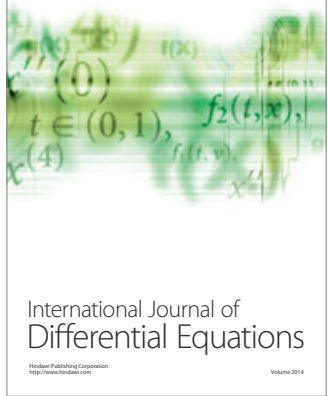
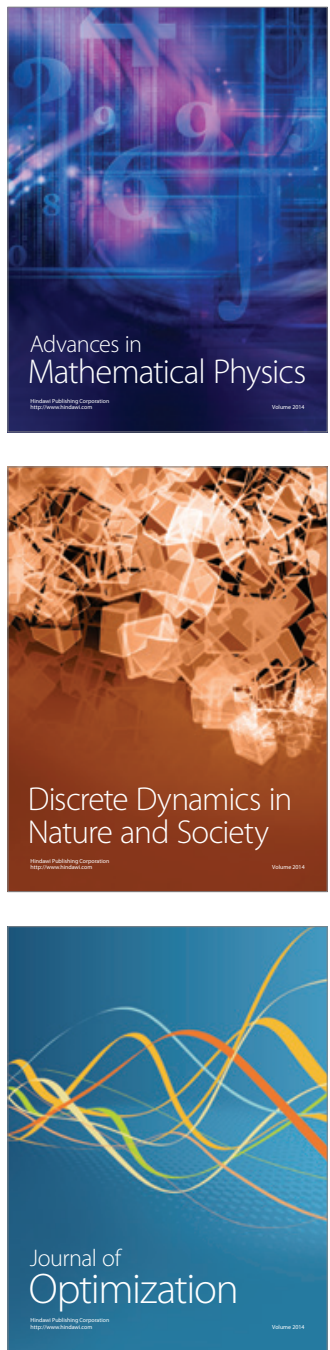